\title{
Bioprosthesis in the Mitral Position: Bovine Pericardial versus Porcine Xenograft
}

\author{
Dong Youb Han, M.D. ${ }^{1}$, Sung Jun Park, M.D. ${ }^{2}$, Ho Jin Kim, M.D. ', Sung-Ho Jung, M.D., Ph.D. ', Suk Jung Choo, M.D., Ph.D.', \\ Cheol Hyun Chung, M.D., Ph.D. ', Jae Won Lee, M.D., Ph.D. ', Joon Bum Kim, M.D., Ph.D.' \\ 'Department of Thoracic and Cardiovascular Surgery, Asan Medical Center, University of Ulsan College of Medicine; ${ }^{2}$ Department of Thoracic and Cardiovascular \\ Surgery, Chung-Ang University Hospital, Seoul, Korea
}

\section{ARTICLE INFO}

Received September 8, 2021

Revised December 9, 2021

Accepted December 14, 2021

Corresponding author

Joon Bum Kim

Tel $82-2-3010-3580$

Fax 82-2-3010-6966

E-mail jbkim1975@amc.seoul.kr

ORCID

https://orcid.org/0000-0001-5801-2395
Background: While the use of bioprosthetic valves for mitral valve replacement (MVR) is increasing, very few studies have compared bovine pericardial and porcine valves in the mitral position to help guide bioprosthetic selection.

Methods: In the present study, patients who underwent MVR using bovine pericardial valves were compared with those who underwent MVR with porcine bioprostheses between January 1996 and July 2018. Those with prior MVR, infective endocarditis, congenital mitral valve disease, or ischemic mitral regurgitation were excluded. The primary outcomes were structural valve deterioration (SVD) and mitral valve reoperation from any cause, and death was regarded as a competing risk. Competing risk analysis and propensity score-matching were used for comparisons.

Results: Among the 388 patients enrolled, pericardial and porcine bioprostheses were implanted in 217 (55.9\%) and 171 (44.1\%), respectively. Propensity score-matching yielded 122 pairs of patients that were well-balanced for all baseline covariates. No significant differences were observed between the groups in unadjusted $(p=0.09)$ and adjusted overall survival (hazard ratio [HR], 1.13; 95\% confidence interval [Cl], 0.72-1.76; $p=0.60$ ). Competing risk analysis revealed no significant differences in the risks of mitral reoperation $(H R, 1.07 ; 95 \% \mathrm{Cl}, 0.50-2.27 ; p=0.86)$ and development of SVD $(H R, 1.57 ; 95 \% \mathrm{Cl}, 0.56-4.36$; $\mathrm{p}=0.39$ ) between the groups. Matched population analysis confirmed similar results regarding reoperation $(\mathrm{HR}, 0.99 ; 95 \% \mathrm{Cl}, 0.40-3.22 ; \mathrm{p}=0.98)$ and $\mathrm{SVD}(\mathrm{HR}, 1.39 ; 95 \% \mathrm{Cl}, 0.41-$ 4.73; $p=0.60$ ).

Conclusion: No significant differences in survival or valve durability were observed between bovine pericardial and porcine bioprosthetic MVR. These findings require further validation through studies with larger sample sizes.

Keywords: Mitral valve replacement, Heart valve bioprosthesis

\section{Introduction}

Bioprosthetic valves have become a standard choice for mitral valve replacement (MVR) in elderly patients with limited life expectancy, as they do not require the recipients to take lifelong anticoagulation medication. A recent large-scale study of registry data showed that patients aged 50 to 69 years who received bioprosthetic MVR had comparable overall survival at 15 years to those who received a mechanical prosthesis [1]. Although the latest American College of Cardiology/American Heart Association guide- lines recommend mechanical valve MVR for patients under 65 years of age who have no contraindication to anticoagulation, the rapid expansion of transcatheter technologies has permitted bioprosthetic surgical valve replacement in younger patients with an expectation of future valve-invalve procedures instead of open surgical reintervention [2]. Given these circumstances, bioprosthetic valves are expected to become more frequently preferred for MVR, mirroring the trend for the aortic valve position.

Valve durability is the main concern with bioprosthetic valves, and the development of structural valve deteriora- 
tion (SVD) is known to be strongly associated with outcomes [3]. Very limited evidence, however, exists directly comparing the durability of the most frequently implanted bioprosthetics in the mitral position: bovine pericardial tissue and porcine xenograft valves. Surgeons therefore have relied on results derived from the data on aortic valve replacement when selecting the type of bioprosthesis in cases of MVR. Thus, comparative studies of bovine pericardial and porcine valves in the mitral position are needed, and the data derived from such studies will be clinically informative and can help guide bioprosthetic valve selection in MVR. In the present study, we sought to compare the long-term performance of bovine pericardial and porcine bioprostheses for surgical MVR using a reasonable sample size and rigorous statistical adjustments.

\section{Methods}

This study was reviewed and approved by the institutional ethics committee/review board of Asan Medical Center (IRB approval no., 2019-0229), which waived the requirement for informed patient consent because of the retrospective nature of the study.

\section{Study population}

In this study, we reviewed adult (aged 18 years or older) patients who received MVR using biologic prostheses with either bovine pericardial or porcine xenograft valves between January 1996 and December 2018 at Asan Medical Center. We excluded those who received concomitant thoracic aortic replacement and those with a history of previous cardiac surgery, infective endocarditis, congenital mitral valve defects, or ischemic mitral regurgitation associated with myocardial infarction. To ensure a large sample size and improve the statistical power, we included patients who underwent MVR with or without concomitant aortic valve replacement, tricuspid valve surgery, ablation of atrial fibrillation, or coronary artery bypass surgery.

\section{Outcomes}

The primary outcomes of interest were cumulative incidence of mitral valve reoperation from any cause and development of SVD. Death was regarded as a competing risk against the primary outcomes. Patient mortality status was obtained from the National Health Insurance Service, which reflects the status on a real-time basis.

\section{Surgical procedure}

Each MVR procedure was performed through a longitudinal right-sided left atriotomy with either a median sternotomy or a right mini-thoracotomy approach. The valve type, either a bovine pericardial valve or a porcine bioprosthesis, was selected based on the surgeon's preference. The subvalvular apparatus was preserved whenever possible, and the prosthesis was implanted with horizontal mattress pledget-buttressed sutures.

\section{Statistical analysis}

Categorical variables, presented as frequencies and percentages, were compared using the chi-square test or the Fisher exact test. Continuous variables were expressed as mean \pm standard deviation or median with range as appropriate. To reduce the impacts of treatment selection bias and potential confounders in the comparisons of outcomes between the bovine pericardial and porcine groups, we adjusted for the differences in baseline variables with propensity scores (PSs). The PSs were estimated using a logistic regression model incorporating all baseline variables listed in Table 1. PS-matched pairs were created by matching between the sequential and the individual groups on the logit of the PS using calipers of width $\leq 0.20$ of the standard deviation of the logit of the PS. The balance of baseline covariates in the matched cohort was assessed using standardized mean differences; standardized differences of $10 \%$ or lower were regarded as indicating well-balanced matching, and standardized differences of $20 \%$ or lower were regarded as indicating acceptable balance [4].

Kaplan-Meier analyses were used to assess the conditional probability of overall death, and log-rank tests were used to compare inter-group differences. In the matched population, logistic regression and Cox proportional hazards regression were used to compare 30-day mortality and long-term mortality, respectively, between groups. The proportional hazards assumption in the Cox model was assessed using Schoenfeld residuals. The cumulative incidence of reoperation and the development of SVD after the index MVR were compared between valve types, with death as a competing risk. Subdistribution hazards in the matched populations were estimated using the method detailed by Fine and Gray [5].

All reported p-values were 2-sided, and p-values of $<0.05$ were considered to indicate statistical significance. $\mathrm{R}$ software ver. 3.4.0 (The R Foundation for Statistical Computing, Vienna, Austria; http://www.R-project.org/) was used for the statistical analysis. 
Table 1. Baseline characteristics and surgical profiles of patients

\begin{tabular}{|c|c|c|c|c|c|c|c|}
\hline \multirow[b]{2}{*}{ Characteristic } & \multicolumn{3}{|c|}{ Overall cohort (unadjusted) } & \multicolumn{4}{|c|}{ Propensity score-matching cohort } \\
\hline & $\begin{array}{l}\text { Bovine } \\
(n=217)\end{array}$ & $\begin{array}{l}\text { Porcine } \\
(n=171)\end{array}$ & p-value & $\begin{array}{l}\text { Bovine } \\
(n=122)\end{array}$ & $\begin{array}{l}\text { Porcine } \\
(n=122)\end{array}$ & p-value & $\begin{array}{c}\text { SMD } \\
(\%)\end{array}$ \\
\hline Age (yr) & $68.8 \pm 9.9$ & $68.4 \pm 11.0$ & 0.71 & $68.3 \pm 10.5$ & $68.2 \pm 11.2$ & 0.93 & 1.1 \\
\hline Female sex & $145(66.8)$ & $112(65.5)$ & 0.86 & $80(65.6)$ & $79(64.8)$ & 1.00 & 1.7 \\
\hline Body mass index $\left(\mathrm{kg} / \mathrm{m}^{2}\right)$ & $21.9 \pm 3.4$ & $22.3 \pm 3.1$ & 0.21 & $22.3 \pm 2.8$ & $22.0 \pm 2.93$ & 0.57 & 7.2 \\
\hline \multicolumn{8}{|l|}{ Comorbidities } \\
\hline Hypertension & $74(34.1)$ & $65(38.0)$ & 0.49 & $44(36.1)$ & $45(36.9)$ & 1.00 & 1.7 \\
\hline Diabetes mellitus & $40(18.4)$ & $36(21.1)$ & 0.60 & $21(17.2)$ & $25(20.5)$ & 0.62 & 8.4 \\
\hline Hemoglobin (mg/dL) & $12.1 \pm 1.84$ & $12.4 \pm 1.69$ & 0.04 & $12.2 \pm 1.78$ & $12.2 \pm 1.63$ & 0.94 & 1.0 \\
\hline Creatinine (mg/dL) & $1.05 \pm 0.67$ & $1.01 \pm 0.56$ & 0.57 & $1.05 \pm 0.56$ & $1.02 \pm 0.63$ & 0.73 & 4.4 \\
\hline eGFR $\left(\mathrm{mL} / \mathrm{min} / 1.73 \mathrm{~m}^{2}\right)$ & $67.9 \pm 22.4$ & $69.0 \pm 18.9$ & 0.57 & $67.1 \pm 20.7$ & $69.8 \pm 19.5$ & 0.29 & 13.0 \\
\hline End-stage renal disease & $3(1.4)$ & $2(1.2)$ & 0.99 & $2(1.6)$ & $2(1.6)$ & 1.00 & $<0.1$ \\
\hline Chronic lung disease & $7(3.2)$ & $6(3.5)$ & 0.99 & $3(2.5)$ & $3(2.5)$ & 1.00 & $<0.1$ \\
\hline History of cerebrovascular accident & $15(6.9)$ & $22(12.9)$ & 0.071 & $8(6.6)$ & $9(7.4)$ & 1.00 & 3.2 \\
\hline History of coronary artery disease & $6(2.8)$ & $4(2.3)$ & 0.99 & $4(3.3)$ & $3(2.5)$ & 1.00 & 4.9 \\
\hline Peripheral vascular disease & $12(5.5)$ & $12(7.0)$ & 0.69 & $5(4.1)$ & $8(6.6)$ & 0.56 & 11.0 \\
\hline Atrial fibrillation & $104(47.9)$ & $90(52.6)$ & 0.41 & $61(50.0)$ & $64(52.5)$ & 0.79 & 4.9 \\
\hline \multicolumn{8}{|l|}{ Echocardiographic data } \\
\hline LV ejection fraction (\%) & $56.7 \pm 9.7$ & $56.4 \pm 10.4$ & 0.81 & $56.0 \pm 10.3$ & $56.3 \pm 10.5$ & 0.79 & 4.5 \\
\hline LV end-systolic dimension (mm) & $35.9 \pm 7.9$ & $35.6 \pm 9.2$ & 0.74 & $36.2 \pm 8.2$ & $35.6 \pm 9.4$ & 0.61 & 6.6 \\
\hline LV end-diastolic dimension (mm) & $53.7 \pm 8.9$ & $53.2 \pm 9.3$ & 0.59 & $53.9 \pm 9.3$ & $53.4 \pm 9.6$ & 0.68 & 5.3 \\
\hline Left atrium diameter (mm) & $56.0 \pm 11.3$ & $56.7 \pm 10.9$ & 0.51 & $56.4 \pm 11.7$ & $56.8 \pm 11.2$ & 0.78 & 3.5 \\
\hline Peak TR pressure gradient (mm Hg) & $40.2 \pm 15.7$ & $40.9 \pm 15.8$ & 0.64 & $41.0 \pm 15.5$ & $40.8 \pm 15.9$ & 0.9 & 1.6 \\
\hline $\mathrm{TR} \geq$ grade 3 & $34(44.8)$ & $61(46.9)$ & 0.762 & $44(36.1)$ & $53(43.4)$ & 0.22 & 18.0 \\
\hline \multicolumn{8}{|l|}{ Concomitant surgical procedure } \\
\hline Aortic valve replacement & $79(36.4)$ & $41(24.0)$ & 0.012 & $36(29.5)$ & $34(27.9)$ & 0.887 & 3.6 \\
\hline Coronary arterial bypass grafting & $30(13.8)$ & $20(11.7)$ & 0.63 & $19(15.6)$ & $18(14.8)$ & 1 & 2.3 \\
\hline Maze procedure & $87(40.1)$ & $88(51.5)$ & 0.033 & $55(45.1)$ & $58(47.5)$ & 0.79 & 4.9 \\
\hline Tricuspid valve replacement & $9(4.1)$ & $2(1.2)$ & 0.14 & $3(2.5)$ & $1(0.8)$ & 0.61 & 1.3 \\
\hline Tricuspid valve repair & $93(42.9)$ & $85(49.7)$ & 0.21 & $53(43.4)$ & $64(52.5)$ & 0.2 & 18.1 \\
\hline
\end{tabular}

Values are presented as mean \pm standard deviation or number (\%).

SMD, standardized mean difference; eGFR, estimated glomerular filtration rate; LV, left ventricular; TR, tricuspid regurgitation.

\section{Results}

\section{Patients}

A total of 2,431 adult patients who received surgical MVR between January 1996 and December 2018 were identified in the cardiac surgical database of Asan Medical Center, Seoul, Korea, in which all patients who received cardiac surgery were prospectively registered. After applying the exclusion criteria, among 561 patients with bioprosthetic valves, data from 388 patients (age, $68.6 \pm 10.4$ years; 257 women) who underwent MVR using bovine pericardial $(n=217)$ or porcine xenograft $(n=171)$ valves were analyzed (Fig. 1).

The baseline and surgical profiles of the patients are shown in Table 1 (left columns). The baseline characteristics of the 2 groups of patients were statistically similar for

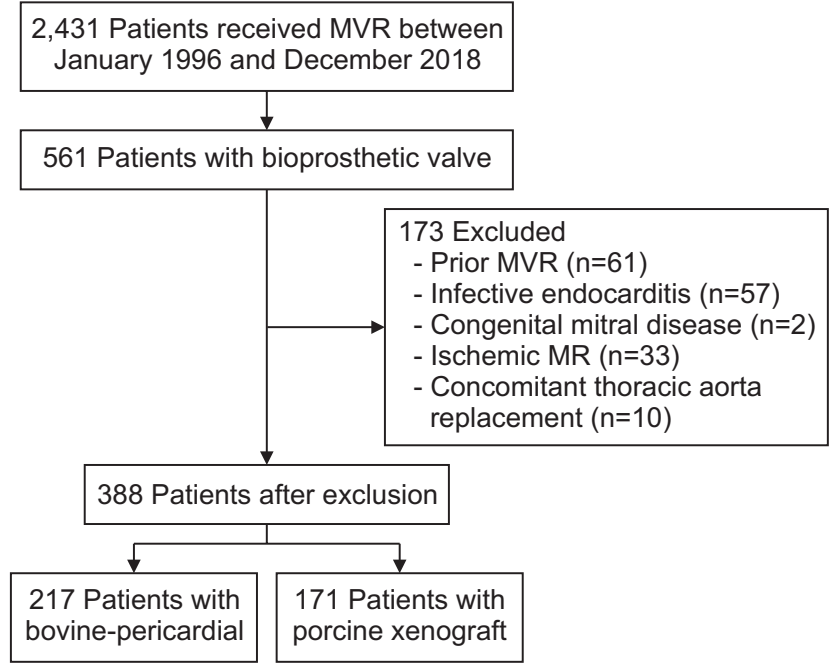

Fig. 1. Patient selection flowchart. MVR, mitral valve replacement; MR, mitral regurgitation. 
almost all variables. Concomitant aortic valve replacements were more frequently performed in the bovine pericardial group than in the porcine group $(\mathrm{p}=0.012)$, while concomitant maze procedures were more frequently performed in the porcine group ( $\mathrm{p}=0.033)$. All of the bovine pericardial prosthetics implanted were Edwards Lifesciences (Edwards Lifesciences Inc., Irvine, CA, USA), Perimount (Edwards Lifesciences Inc.), or Magna Mitral Ease (Edwards Lifesciences Inc.) pericardial valves. The most frequently implanted porcine valve was the Medtronic Hancock II valve (Medtronic Inc., Minneapolis, MN, USA) (Table 2).

The PS-matching model yielded a total of 122 matched pairs of patients who underwent MVR using bovine pericardial or porcine bioprosthetic valves (Table 1, right columns). The 2 groups of patients in the matched population were largely well-balanced, with a standardized mean difference of lower than $20 \%$ for all variables.

\section{Overall survival}

Early death (within 30 days) occurred in 16 of 217 pa- tients $(7.4 \%)$ in the bovine pericardial and 6 of 171 patients (3.5\%) in the porcine group ( $\mathrm{p}=0.16)$. The risk of early death did not differ significantly between the 2 groups (odds ratio [OR], $0.58 ; 95 \%$ confidence interval [CI], 0.19 to 1.61; $\mathrm{p}=0.31$ ) after PS matching.

Over a median follow-up period of 42.7 months (interquartile range, 14.1-106.1) among surviving patients, 79 (7.2\%/patient-year) and 40 (4.9\%/patient-year) patients died in the bovine pericardial and porcine groups, respectively.

No significant difference was observed between the 2 groups in overall survival $(\mathrm{p}=0.09)$ (Fig. $2 \mathrm{~A})$. After adjustment using the PS matching method, the overall risk of death did not differ significantly between the groups (hazard ratio $[\mathrm{HR}$ ], $0.94 ; 95 \% \mathrm{CI}, 0.60$ to $1.46 ; \mathrm{p}=0.80$ ) (Fig. $2 \mathrm{~B}$ ).

\section{Mitral reoperation}

The competing risk regression revealed no significant difference in the risk of mitral reoperation (HR, 1.07; 95\% CI, 0.5-2.27) $(\mathrm{p}=0.86)$ when death was regarded as a competitive event (Fig. 3A). The adjusted risks of reoperation were also not statistically different (HR, 0.99; 95\% CI, 0.4-

Table 2. Number and size distribution of bioprostheses implanted over the study period

\begin{tabular}{|c|c|c|c|c|c|}
\hline \multirow{2}{*}{$\begin{array}{l}\text { Bioprosthesis size } \\
\text { distribution }(\mathrm{mm})\end{array}$} & \multicolumn{2}{|c|}{ Bovine pericardial bioprosthesis $(n=217$ ) } & \multicolumn{3}{|c|}{ Porcine bioprosthesis $(n=171)$} \\
\hline & Perimount $(n=143)$ & Magna mitral ease $(n=74)$ & Hancock II (n=134) & Epic $(n=8)$ & Biocor $(n=29)$ \\
\hline 23 & 1 & 0 & 0 & 0 & 0 \\
\hline 25 & 19 & 9 & 4 & 0 & 0 \\
\hline 27 & 44 & 29 & 49 & 3 & 7 \\
\hline 29 & 54 & 24 & 48 & 2 & 8 \\
\hline 31 & 19 & 7 & 23 & 3 & 13 \\
\hline 33 & 6 & 5 & 10 & 0 & 0 \\
\hline 35 & 0 & 0 & 0 & 0 & 1 \\
\hline
\end{tabular}

A

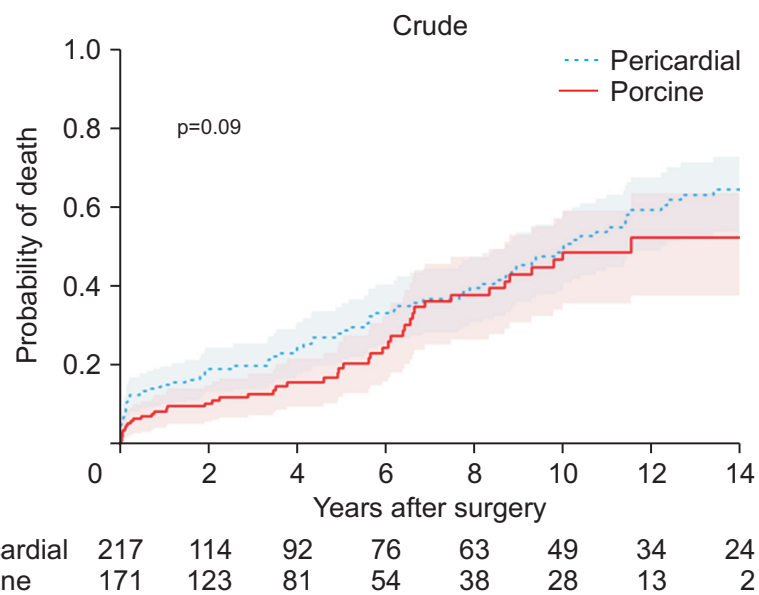

B

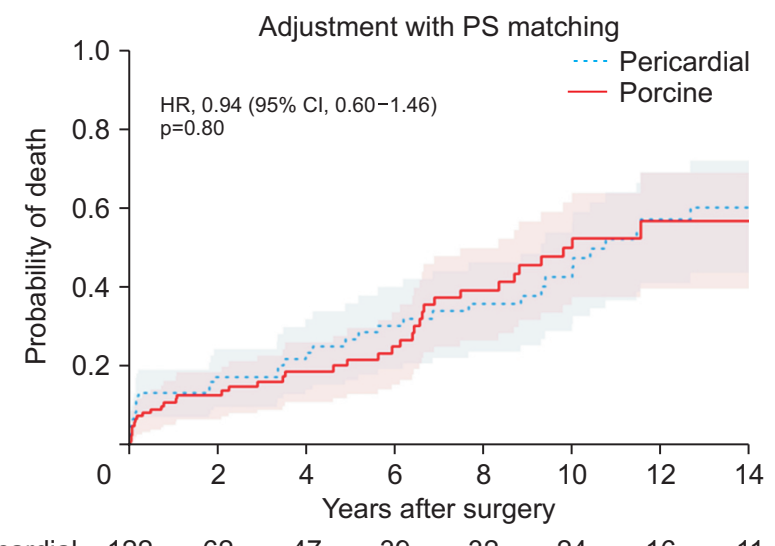

$\begin{array}{lllllllll}\text { Pericardial } & 122 & 62 & 47 & 39 & 32 & 24 & 16 & 11\end{array}$

$\begin{array}{lllllllll}\text { Porcine } & 122 & 83 & 60 & 44 & 31 & 22 & 10 & 2\end{array}$

Fig. 2. Probability of death (A) before and (B) after propensity score (PS) matching. HR, hazard ratio; Cl, confidence interval. 
2.44; $\mathrm{p}=0.98$ ) after PS matching (Fig. 3B). The 5-year, 10 year, and 15-year cumulative rates of reoperation in the entire cohort were $1.6 \%, 14.8 \%$, and $51.5 \%$, respectively (Table 3).

No significant difference was found in the risk of development of SVD between groups both before (HR, 1.57; 95\% CI, 0.56-4.36; $\mathrm{p}=0.39)$ and after matching (HR, 1.39; 95\% CI, $0.41-4.73 ; \mathrm{p}=0.60)$. The cumulative incidence of SVD is illustrated in Fig. 3C and D.

The causes of mitral reoperations are detailed in Table 4. Before matching, reoperation due to calcified thickening was more frequent in the bovine group than in the porcine group. However, the causes of reoperation were not significantly different between groups for both SVD and nonSVD causes in the matched population.

Of those 5 cases of SVD in the porcine group, degenerative changes such as valve leaflet thickening or calcification developed in only 1 patient, while leaflet tear or perforation occurred in the remaining 4 patients. All leaflet tears or perforations occurred in patients treated with porcine Epic (Abbott, Chicago, IL, USA) or Biocor (Abbott) valves.

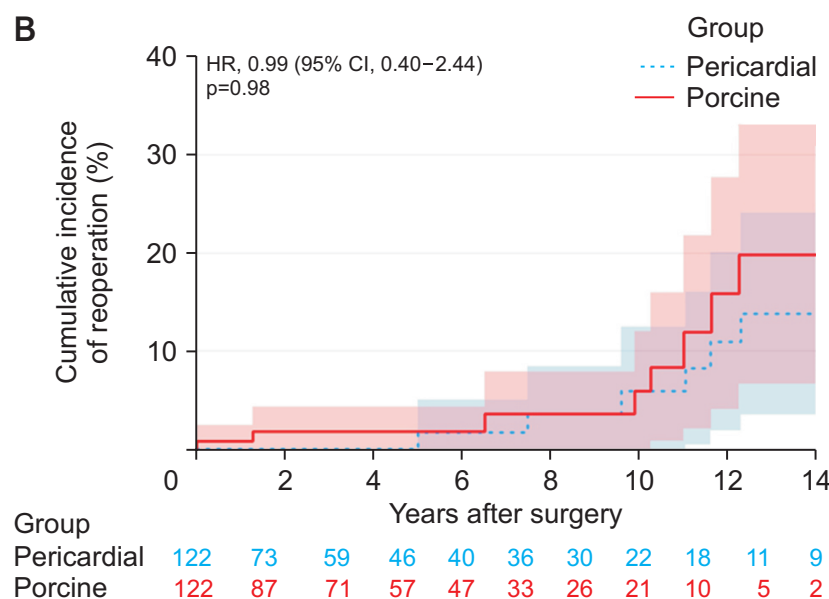

A

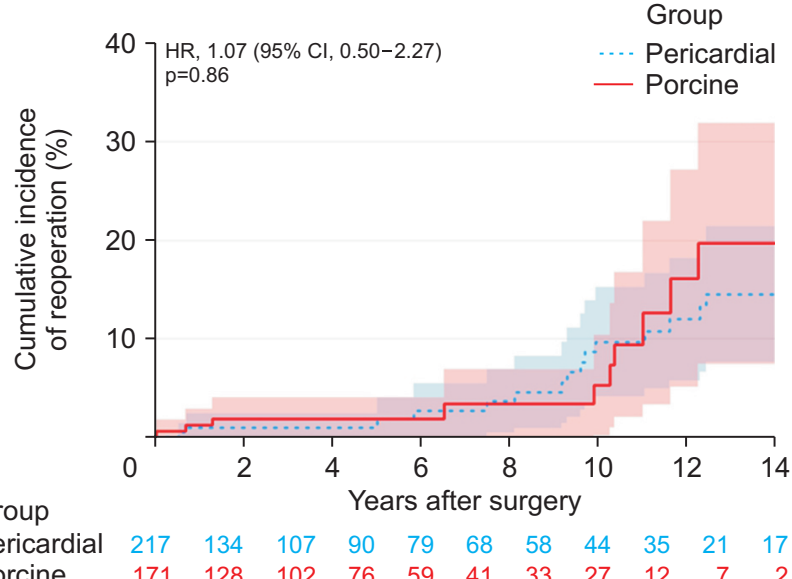

C

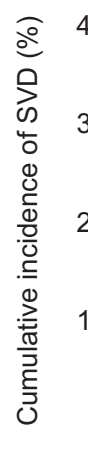

Group

$\begin{array}{lllllllllllr}\text { Porcine } & 171 & 128 & 102 & 76 & 59 & 41 & 33 & 27 & 12 & 7 & 2\end{array}$

Group

Pericardial

- Porcine $\begin{array}{llllllllllll}\text { Pericardial } & 217 & 134 & 107 & 90 & 79 & 68 & 58 & 44 & 35 & 21 & 17\end{array}$

Fig. 3. (A) Unadjusted and (B) adjusted cumulative incidence of mitral reoperation. (C) Unadjusted and (D) adjusted cumulative incidence of structural valve deterioration (SVD). $\mathrm{HR}$, hazard ratio; $\mathrm{Cl}$, confidence interval.

Table 3. Cumulative event rate of reoperation

\begin{tabular}{lccc}
\hline \multirow{2}{*}{ Variable } & \multicolumn{3}{c}{ Cumulative event rate \% $(95 \%$ confidence interval $)$} \\
\cline { 2 - 4 } & Overall $(\mathrm{n}=338)$ & Bovine pericardial $(\mathrm{n}=217)$ & Porcine $(\mathrm{n}=171)$ \\
\hline At 5 years & $1.6(0.2-3.0)$ & $1.2(0.0-2.8)$ & $2.0(0.0-4.3)$ \\
At 10 years & $14.8(6.9-21.9)$ & $18.4(7.2-28.3)$ & $8.5(0.0-17.2)$ \\
At 15 years & $51.5(21.5-70.1)$ & $52.9(16.3-73.4)$ & $39.1(9.9-58.9)$ \\
\hline
\end{tabular}


Table 4. Causes of mitral reoperation

\begin{tabular}{|c|c|c|c|c|c|c|}
\hline \multirow{2}{*}{ Variable } & \multicolumn{3}{|c|}{ Overall cohort (unadjusted) } & \multicolumn{3}{|c|}{ Propensity score-matching cohort } \\
\hline & Bovine $(n=217)$ & Porcine $(n=171)$ & p-value & Bovine $(n=122)$ & Porcine $(n=122)$ & $\mathrm{p}$-value \\
\hline Reoperation cause & $20(9.2)$ & $11(6.4)$ & & $9(7.4)$ & $9(7.4)$ & $>0.99$ \\
\hline SVD & $13(6.0)$ & $5(2.9)$ & 0.24 & $6(4.9)$ & $5(4.1)$ & 0.75 \\
\hline Leaflet tear/perforation & $2(0.9)$ & $4(2.3)$ & 0.48 & $2(1.6)$ & $4(3.3)$ & 0.68 \\
\hline Thickening/calcification & $11(5.1)$ & $1(0.6)$ & 0.03 & $4(3.3)$ & 0 & 0.13 \\
\hline Non-SVD & $7(3.2)$ & $6(3.5)$ & 0.95 & $3(2.5)$ & $4(3.3)$ & $>0.99$ \\
\hline Infective endocarditis & $3(1.4)$ & $2(1.2)$ & $>0.99$ & $2(1.6)$ & $1(0.8)$ & $>0.99$ \\
\hline Pannus formation & $3(1.4)$ & $2(1.2)$ & $>0.99$ & $1(0.8)$ & $1(0.8)$ & $>0.99$ \\
\hline Paravalvular leakage & $1(0.5)$ & $2(1.2)$ & 0.84 & 0 & $2(1.6)$ & 0.48 \\
\hline
\end{tabular}

Values are presented as number (\%).

SVD, structural valve deterioration.

\section{Edwards Lifesciences pericardial valves versus Medtronic Hancock II porcine valves}

Comparisons were also made between the most frequently implanted models in each group: Edwards Lifesciences pericardial valves and Medtronic Hancock II porcine valves. No significant difference in overall survival $(\mathrm{p}=0.09)$ was observed between the 2 groups (Supplementary Fig. 1). Additionally, no statistically significant differences were found in the cumulative risks of mitral reoperation (HR, 1.39; 95\% CI, 0.53-3.67; $\mathrm{p}=0.50$ ) and development of SVD (HR, 5.01; 95\% CI, 0.65-38.8; p=0.12) (Supplementary Fig. 2).

\section{Discussion}

With the advent of transcatheter aortic valve replacement, both the interest in and demand for biologic prosthetic aortic valves have exponentially increased, concurrent with timely modifications in age for prosthetic valve selection under current practice guidelines [2]. This increasingly prevalent use of biologic prosthetic valves will not be limited to the aortic position, and expansion to the mitral valves is anticipated as rapid advancements are made in transcatheter technology. However, although biologic prosthetic valves have the important clinical advantage of not requiring a lifelong anticoagulation regimen, these valves involve significant limitations in durability, which is closely associated with patient outcomes [3]. In bioprosthetic valves, SVD develops with time, during which reoperation is occasionally required.

Unlike valve replacements in the aortic position, the performance of implanted bioprosthetic mitral valves has not been evaluated in depth based on valve material. The performance of prostheses is clinically significant in aortic valve replacement, particularly when small valves are implanted, because valve hemodynamics contribute to patients-prosthesis mismatch [6]. It is generally accepted that the clinical relevance of patient-prosthesis mismatch is less significant in the mitral than in the aortic position; the performance of prostheses in this position has thus not been a concern for researchers. Given such limited evidence derived from scientific data in the mitral position, surgeons have relied on research focused on aortic valves for MVR. This is based on a belief that the outcomes associated with bioprosthetic valves in the mitral position will resemble those of aortic valves, even though the hemodynamic profiles completely differ between those cardiac valves. However, as the use of biologic valves in the mitral position is expected to exponentially increase, as occurred with the aortic valve, observational research on the outcomes of bioprosthetic valves in the mitral position will be informative to clinicians. Although survival and late outcomes by valve material in the mitral position have been reported in previous studies [7,8], few reports exist evaluating the results of bioprosthetic valves, including reoperation and SVD, regardless of specific valve type.

In the present study, no significant difference was observed in overall survival for 15 years between patients who received MVR using bovine pericardial versus porcine xenograft valves. Similar results were replicated in the PSmatched population. The competing risk analysis also revealed no significant differences in the risks of mitral reoperation and development of SVD according to bioprosthetic valve material. While both groups showed similar risks of reoperation, that risk remained under $7 \%$ until 9 years after the index surgery; thereafter, the risk of reoperation increased sharply, with rates of $1.6 \%$ at 5 years, $6.4 \%$ at 9 years, $14.8 \%$ at 10 years, and $51.5 \%$ at 15 years after the index surgery. These findings show that the risks of reopera- 
tion increase dramatically between 9 and 10 years after surgery.

In the sub-analysis of the most frequently implanted models in each group, the Edwards Lifesciences Perimount or Magna Mitral Ease valves were compared against the Medtronic Hancock II. The risk of SVD appeared higher in the Edwards Lifesciences pericardial valves, although this difference failed to reach statistical significance because of the small sample size. Only 1 case of SVD was observed, at 11 years after surgery, with the Hancock II valve. Further studies with longer follow-up periods and larger sample sizes will be required to draw a clear conclusion.

It is commonly believed that pericardial valves are resistant to shear stress but that porcine valves are susceptible to shear stress and subsequent leaflet tears. However, our study demonstrates no statistically significant difference in the occurrence of SVD depending on the valve material. Notably, all cases of porcine valve leaflet complications occurred with Saint-Jude Epic or Biocor valves, at what seems an unacceptably high rate (1.92\%/patient-year, 4/37) considering the limited use of this valve. However, according to Jawad et al. [9], Epic valves in the mitral position showed acceptable rates of survival and valve-related complications or reoperation at midterm follow-up. Further investigation seems to be warranted to examine the long-term safety of these valves.

\section{Limitations}

This study is subject to the limitations inherent to retrospective studies with an observational design. Although a high level of adjustment was performed to reduce the selection bias between the groups, residual selection bias may have existed due to unmeasured confounding variables. In the present study, we included patients with multiple valve replacements to improve the statistical power. Although the inclusion of patients receiving concomitant aortic valve replacement is the main limitation of the present study, we believe that rigorous adjustment was accomplished by PS matching. However, the comparison of Edward and Medtronic valves may have been biased by the lack of adjustment for preoperative variables. Lastly, since the clinical follow-up duration for analysis of bioprosthesis longevity was relatively short, further investigation with long-term clinical follow-up is needed.

\section{Conclusions}

In bioprosthetic valve replacements in the mitral posi- tion, bovine pericardial and porcine xenograft valves showed comparable survival and valve durability. These findings require further validation through studies with larger sample sizes.

\section{Conflict of interest}

No potential conflict of interest relevant to this article was reported.

\section{ORCID}

Dong Youb Han: https://orcid.org/0000-0003-0924-5197 Sung Jun Park: https://orcid.org/0000-0002-0244-062X Ho Jin Kim: https://orcid.org/0000-0002-0809-2240 Sung-Ho Jung: https://orcid.org/0000-0002-3699-0312 Suk Jung Choo: https://orcid.org/0000-0003-4291-302X Cheol Hyun Chung: https://orcid.org/0000-0001-8981-6011 Jae Won Lee: https://orcid.org/0000-0003-0751-2458 Joon Bum Kim: https://orcid.org/0000-0001-5801-2395

\section{Supplementary materials}

Supplementary materials can be found via https://doi. org/10.5090/jcs.21.103. Supplementary Fig. 1. Probability of death over time for patients treated with the Perimount pericardial valve versus the Hancock II porcine valve. Supplementary Fig. 2. (A) Cumulative incidence of mitral reoperation and (B) cumulative incidence of structural valve deterioration (SVD) over time for patients treated with pericardial versus porcine valves.

\section{References}

1. Chikwe J, Chiang YP, Egorova NN, Itagaki S, Adams DH. Survival and outcomes following bioprosthetic vs mechanical mitral valve replacement in patients aged 50 to 69 years. JAMA 2015;313:1435-42.

2. Otto CM, Nishimura RA, Bonow RO, et al. 2020 ACC/AHA guideline for the management of patients with valvular heart disease: executive summary: a report of the American College of Cardiology/ American Heart Association Joint Committee on Clinical Practice Guidelines. Circulation 2021;143:e35-71.

3. Andreas M, Wallner S, Ruetzler K, et al. Comparable long-term results for porcine and pericardial prostheses after isolated aortic valve replacement. Eur J Cardiothorac Surg 2015;48:557-61.

4. Silber JH, Rosenbaum PR, Trudeau ME, et al. Multivariate matching and bias reduction in the surgical outcomes study. Med Care 2001; 39:1048-64.

5. Fine JP, Gray RJ. A proportional hazards model for the subdistribu- 
tion of a competing risk. J Am Stat Assoc 1999;94:496-509.

6. Borger MA, Nette AF, Maganti M, Feindel CM. Carpentier-Edwards Perimount Magna valve versus Medtronic Hancock II: a matched hemodynamic comparison. Ann Thorac Surg 2007;83:2054-8.

7. Tsubota H, Sakaguchi G, Arakaki R, Marui A. Comparison of porcine versus bovine pericardial bioprosthesis in the mitral position. $\mathrm{J}$ Card Surg 2021;36:2776-83.
8. Kim W, Hwang HY, Kang Y, et al. Comparative analysis of structural valve deterioration and long-term clinical outcomes after bovine pericardial versus porcine bioprosthetic mitral valve replacement. J Thorac Dis 2021;13:3969-78.

9. Jawad K, Lehmann S, Koziarz A, et al. Midterm results after St Jude Medical Epic porcine xenograft for aortic, mitral, and double valve replacement. J Card Surg 2020;35:1769-77. 